\title{
Prévention \\ des déficiences nutritionnelles \\ chez les personnes sans-abri : \\ intérêt d'un aliment de rue enrichi
}

Nicole DARMON

André BRIEND 


\title{
NICOLE DARMON *, ANDRÉ BRIEND **
}

\section{A fortified street food to prevent nutrient deficiencies in bomeless}

\begin{abstract}
Summary - Existing food aid programs have failed to prevent insufficient dietary intakes and micronutrient deficiencies in the population of homeless in Paris. To improve these programs, we designed a fortified food easy to consume in the street. Its economic interest was estimated by linear programming. We further tested its acceptability among the target population. A chocolate-flavoured spread naturally rich in potassium and $n-3$ fatty acids was fortified with calcium, zinc, vitamins $C, D, E, B_{12}$, thiamine, niacin and folic acid. This spread presents multiple advantages for bomeless nutrition: resistance to bacterial contamination, viscosity adapted to people having limited chewing capacity, bigh energy density, and high nutritional quality/price ratio. Indeed, adding 1 packet of Vitapoche ${ }^{\circledR}$ to a classical food basket increases, for a low cost (21 eurocents/packet), the energy content of the basket, and significantly improves its nutritional quality. This represents an economic advantage for the donor, as well as for the food aid recipient. The acceptability study was conducted in eight sites providing food aid to homeless. During two weeks in each site, the fortified food was systematically proposed (one packet/day) to each individual visiting the site. The evaluation of this new food, named Vitapoche ${ }^{\circledR}$, showed that homeless would consume the fortified food often or daily, if available. Including fortified street foods in existing food aid programs for homeless is a practical and economic way to belp prevent nutritional deficiencies in this population.
\end{abstract}

Key-words : homeless, fortified food, nutrient deficiencies, economic value, food aid

\section{Prévention des déficiences chez les personnes sans-abri : intérêt d'un aliment de rue enrichi}

Résumé - Pour mieux prévenir la dénutrition et les déficiences vitaminiques et minérales chez les personnes en situation d'exclusion, nous avons mis au point un aliment enrichi facile à consommer dans la rue : le Vitapoche ${ }^{\circledR}$. Il s'agit d'une pâte chocolatée résistante à la contamination bactérienne, emballée dans un sachet hermétique. Cette pâte est naturellement riche en potassium et en acides gras oméga-3. Elle a été enrichie en calcium, zinc et vitamines C, B1, B9, PP, B12, E et D, afin que chaque sachet $(70 \mathrm{~g}, 377 \mathrm{kcal})$ apporte ces nutriments en quantité nécessaire pour la journée. Outre son intérêt nutritionnel évident, le Vitapoche ${ }^{\circledR}$ présente aussi un double intérêt économique, pour le donateur et pour le bénéficiaire. Une étude d'acceptabilité menée au sein de la population cible à Paris a montré que deux personnes sur trois consommeraient régulièrement du Vitapoche ${ }^{\circledR}$ si elles pouvaient se le procurer facilement. Le Vitapoche ${ }^{\circledR}$ n'est pas un substitut de repas. Il n'est pas destiné à remplacer l'aide alimentaire déjà existante, mais il la complète, afin d'en optimiser la qualité nutritionnelle.

Mots-clés : personnes sans-abri, aliments enrichis, déficiences nutritionnelles, intérêt économique, aide alimentaire

* UMR Nutrition bumaine INSERM U476/INRA 1260, Faculté de médecine de la Timone, 27 boulevard Jean-Moulin, 13385 Marseille cedex 05

e-mail : Nicole.Darmon@medecine.univ-mrs.fr

** IRD, Département Sociétés et santé, 213 rue La Fayette, 75480 Paris cedex

Nous remercions la Direction générale de la Santé pour son soutien financier, ainsi que toutes les équipes qui ont contribué à la réalisation de ce projet: Centres d'hébergement d'urgence du bd Ney, de Montrouge et de la Mie de Pain, Coordination pour l'accueil des familles demandeuses d'asile, Halte des Amis de la rue/ Espace 15-25, Espaces Solidarité Insertion de la Mie de Pain et du $10^{\mathrm{e}}$ arrondissement et équipes mobiles d'aide du SAMU social de Paris. Nous remercions tout particulièrement le $\mathrm{D}^{\mathrm{r}}$ Michel Chauliac (DGS, responsable du PNNS) pour son soutien stratégique. 


\section{Des apports alimentaires insuffisants malgré des besoins nutritionnels augmentés}

Privée de domicile, une personne est souvent contrainte de sauter des repas et de consommer des aliments froids dans la rue. Des études montrent que le repas du midi est le plus difficile à assurer (Brousse, 2002; Marpsat, 1999) et que ce manque de nourriture est principalement dû à un manque d'argent (Darmon et al., 2000 ; Malmauret et al., 2002). Pourtant, la dureté des conditions de vie à la rue (importante activité physique liée à la marche, manque de sommeil, exposition à des températures extrêmes...) augmente probablement les besoins énergétiques et devrait être accompagnée d'une augmentation, non d'une diminution, des apports énergétiques. De même, les consommations excessives de tabac et d'alcool, fréquemment observées chez les personnes privées de domicile, diminuent l'absorption de nombreux micronutriments et augmentent les besoins en antioxydants. Ainsi, les apports en vitamines et minéraux, vitamine C (Cross et Halliwell, 1993), zinc (Lecomte et al., 1994), vitamines B (Cook et al., 1998), calcium et vitamine D (Kanis et al., 1999) en particulier, devraient être augmentés en cas de consommation d'alcool et de tabac, et non pas diminués, comme on l'observe chez les personnes sans-abri.

\section{Une prévention nutritionnelle nécessaire}

Nombreuses sont les études qui mettent en évidence une fréquence élevée de malnutrition et de sub-carences vitaminiques et minérales (Darnton-Hill et Truswell, 1990 ; Gelberg et al., 1995; Langnase et Muller, 2001; Laven et Brown, 1985; Luder et al., 1989; Rushton et Wheeler, 1993 ; Silliman et al., 1998; Wolgemuth et al., 1992) chez les personnes privées de domicile. Des cas de scorbut (carence sévère en vitamine C) (Fain et Thomas, 1997), d'encéphalopathie de Wernicke (carence sévère en vitamine B1 associée à l'alcoolisme) (Darnton-Hill et Truswell, 1990) et de pellagre (carence sévère en vitamine PP) (Kertesz, 2001) ont même été décrits.

Des études menées récemment en France confirment que le statut nutritionnel des personnes sans-abri est fortement dégradé (Cadau et al., 2001; Darmon, 2001b ; Darmon et al., 2002 ; Malmauret et al., 2002). Une première enquête menée par notre équipe auprès de personnes accueillies dans un centre d'hébergement d'urgence à Paris a mis en évidence une forte prévalence de déficit pondéral et de faibles niveaux d'apports énergétiques dans cette population. Le tableau 1 indique que les apports moyens ou médians en vitamines $\mathrm{C}, \mathrm{D}, \mathrm{B} 1, \mathrm{~B}$,, $\mathrm{PP}$, calcium, zinc et potassium sont inférieurs aux apports nutritionnels conseillés pour les adultes de sexe masculin (Martin, 2001), suggérant un risque de déficiences nutritionnelles dans la population des personnes sans-abri. Ce risque a été confirmé dans le cas de la vitamine $C$ par une étude menée par le SAMU social auprès de personnes extrêmement désocialisées: $95 \%$ des personnes présentaient une déficience biologique en vitamine $C$, avec des valeurs plasmatiques parfois proches des seuils scorbutiques (Malmauret et al., 2002). Dans les deux études, les apports énergétiques hors alcool étaient faibles (en moyenne $2032 \mathrm{kcal} / \mathrm{j}$ pour les hommes accueillis en centre d'hébergement d'urgence), voire très faibles dans l'étude du SAMU social (en moyenne $1200 \mathrm{kcal} / \mathrm{j}$ ). 
Tableau 1. Apports nutritionnels d'hommes hébergés dans un centre d'hébergement d'urgence à Paris (Darmon, 2001)

\begin{tabular}{|c|c|c|c|}
\hline & Moyenne & Médiane & $\begin{array}{l}\text { Apport nutritionnel } \\
\text { conseillé } \\
\text { (Martin, 2001) }\end{array}$ \\
\hline Energie totale hors alcool $(\mathrm{kcal} / \mathrm{j})$ & $2032(763)^{*}$ & $1930 *$ & 2700 \\
\hline Protéines (\% énergie totale) & $16,8(3,7)$ & 16,7 & $>12 \%$ et $\leq 18 \%$ \\
\hline Lipides (\% énergie totale) & $31,9(7,7)$ & 31,6 & $\leq 33 \%$ \\
\hline Glucides (\% énergie totale) & $51,3(8,6)$ & 51,9 & $\geq 45 \%$ \\
\hline Calcium $(\mathrm{mg} / \mathrm{j})$ & $721(296) *$ & $670 *$ & 900 \\
\hline Potassium (mg/j) & $2941(1188)$ & 2801 & 3100 \\
\hline $\operatorname{Fer}(\mathrm{mg} / \mathrm{j})$ & $14,6(8,1)$ & 13,2 & 9 \\
\hline $\operatorname{Zinc}(\mathrm{mg} / \mathrm{j})$ & $9,50(3,55) *$ & $9,23 *$ & 12 \\
\hline Vitamine Bl (mg/j) & $0,93(0,37) *$ & $0,87 *$ & 1,3 \\
\hline Vitamine B2 (mg/j) & $1,62(1,18)$ & $1,24 *$ & 1,6 \\
\hline Niacine $P P(m g / j)$ & $19,0(9,8)$ & 17,6 & 14 \\
\hline Vitamine B6 (mg/j) & $2,02(1,21)$ & 1,77 & 1,8 \\
\hline Folate, B9 $(\mu \mathrm{g} / \mathrm{j})$ & $259(138) *$ & $234 *$ & 330 \\
\hline Vitamine $\mathrm{C}(\mathrm{mg} / \mathrm{j})$ & $64,8(52,5) *$ & $52,7 *$ & 110 \\
\hline Vitamine $D(\mu g / j)$ & $1,58(2,92) *$ & $0,72 *$ & 5 \\
\hline
\end{tabular}

Les écarts-types figurent entre parenthèses; * significativement plus faible que l'apport conseillé.

Ces déficiences nutritionnelles sont probablement impliquées, au moins en partie, dans les forts taux de morbidité (maladies mentales, pathologies hépatiques et infectieuses et affections bucco-dentaires notamment) (Hwang, 2001; Langnase et Muller, 2001 ; Luder et al., 1990 ; Robertson et Cousineau, 1986 ; Salit et al., 1998 ; Shanks, 1988 ; Takano et al., 1999; Vredevoe et al., 1992) et de mortalité (Babidge et al., 2001; Barrow et al., 1999; Hwang et al., 1998; Hwang, 2000 ; Roy et al., 1998 ; Shaw et Dorling, 1998) observés chez les personnes sans-abri dans tous les pays industrialisés, y compris en France (Lecomte et Mizrahi, 1997 ; Terolle, 2002).

C'est pourquoi la prévention de la dénutrition et des déficiences vitaminiques et minérales chez les personnes en situation d'exclusion a été définie comme un objectif prioritaire de santé publique dans le cadre du Programme national Nutrition Santé, le PNNS (ministère de la Santé, 2001).

\section{Une dépendance importante vis-à-vis de l'aide alimentaire}

Les personnes sans-abri sont les principales utilisatrices des services d'aide alimentaire d'urgence et elles y ont généralement recours de façon quotidienne (Brousse et al., 2002). Ces personnes sont donc dépendantes de l'aide alimentaire. Cependant, les 
repas donnés dans la rue ou dans les foyers ne sont pas toujours équilibrés. Ils manquent souvent de produits frais, fruits et légumes en particulier (Derrickson et al., 1994 ; Laven et Brown, 1985 ; Rushton et Wheeler, 1993). Même quand l'aide alimentaire est de bonne qualité nutritionnelle, le problème de la quantité se pose (Gendron et al., 2003 ; Jacobs-Starkey, 1994). En effet, l'aide alimentaire d'urgence ne couvre au mieux que deux tiers des besoins énergétiques journaliers des personnes sans-abri (Darmon et al., 2001b; Gendron et al., 2003 ; Rushton et Wheeler, 1993). En ce qui concerne le tiers restant, deux cas peuvent être envisagés : soit ce tiers n'est tout simplement pas couvert, soit il l'est, mais les calories manquantes sont apportées par des aliments de qualité nutritionnelle médiocre. Ces aliments, tels que les produits gras et/ou sucrés (type gâteaux secs) ou les boissons sucrées et/ou alcoolisées, apportent des calories, mais pratiquement pas de vitamines, de minéraux ni de fibres. Dans les deux cas, on observe un manque de nutriments essentiels, accompagné dans le premier cas d'un manque d'énergie. Pour prévenir ces déficits d'apport, il faudrait que la quasi-totalité des vitamines, minéraux et acides gras essentiels nécessaires pour la journée soit présente dans la fraction donnée (2/3 des besoins énergétiques), c'est-à-dire que l'aide alimentaire atteigne une densité nutritionnelle (quantité de nutriments/kcal) exceptionnelle, de 1,5 à 2 fois celle d'une alimentation habituelle. Ceci est impossible à réaliser avec des aliments courants et nécessite de faire appel soit à des aliments enrichis soit à des suppléments nutritionnels.

\section{Un usage inadéquat des suppléments nutritionnels}

Le recours à des suppléments nutritionnels, à savoir des formes médicamenteuses de nutriments, n'est pas souhaitable. Il pose en effet des problèmes de faisabilité (nonrespect des prescriptions) et de toxicité potentielle. Ainsi, l'étude du SAMU social a mis en évidence un pourcentage important de personnes présentant des taux sanguins de vitamine B6 anormalement élevés dans la population des personnes vivant sans domicile à Paris (Malmauret et al., 2002). Des supplémentations, ou même des injections, avec des mélanges de vitamines du groupe $\mathrm{B}$ à doses pharmacologiques, pratiquées de façon répétée et non contrôlée par de nombreux services d'urgence pour prévenir les troubles neurologiques associés à l'alcoolisme, expliquent ces résultats paradoxaux. Cette pratique pourrait avoir des effets contraires à ceux attendus, du fait de la neurotoxicité de la vitamine B6 à forte dose (Dalton et Dalton, 1987).

\section{Le Vitapoche ${ }^{\circledR}$ : un aliment enrichi adapté aux besoins nutritionnels des personnes sans-abri}

Dans la pratique, seul un aliment enrichi, facile à consommer dans la rue, de bon rapport qualité/prix, bien accepté par les personnes sans-abri peut permettre d'améliorer de façon notable la qualité nutritionnelle de l'aide alimentaire destinée aux personnes sans-abri. Un tel aliment, le Vitapoche ${ }^{\circledR}$, a été mis au point par notre équipe, en collaboration avec la société Nutriset (www.vitapoche.com), spécialisée dans la conception et la fabrication de produits destinés à l'aide alimentaire d'urgence dans les situations de famine, en Afrique notamment (Diop et al., 2003). Le Vitapoche ${ }^{\circledR}$ se 
présente sous la forme d'une pâte chocolatée enrichie en vitamines et minéraux. Sa formulation est spécifiquement destinée à prévenir les déficiences vitaminiques et minérales suspectées chez les personnes sans-abri. Chaque sachet apporte du calcium, du zinc, des vitamines C, B1, B9, PP, B12, E et D à doses nutritionnelles (voir tableau 2). Les doses ont été choisies de façon à assurer, avec 1 sachet par jour, la couverture des ANC (Apports nutritionnels conseillés) ou des AJR (Apports journaliers recommandés) pour tous les nutriments considérés comme à risque d'être déficitaires dans la ration des personnes sans-abri, sans toutefois dépasser, avec deux sachets par jour, la limite de sécurité. L'aliment n'est pas enrichi en vitamine A, fer ou cuivre, ces nutriments pouvant présenter une hépato-toxicité dans cette population où le risque de développer une cirrhose est particulièrement élevé. Il n'y a pas non plus d'enrichissement en vitamine B6, étant donné la neuro-toxicité de cette vitamine à doses élevées (Dalton et Dalton, 1987). Seules les vitamines B1 et B12 sont présentes en quantité supérieure à l'ANC pour un homme adulte (10 et 4 fois l'ANC respectivement), ceci en vue d'assurer une meilleure prévention des complications hépatiques et neurologiques associées à l'alcoolisme. Ces deux vitamines sont rapidement éliminées en cas d'apports excessifs et ne présentent aucun risque de toxicité. Par ailleurs, la pâte chocolatée qui constitue la base du Vitapoche ${ }^{\circledR}$ est naturellement riche en potassium, magnésium et acides gras essentiels de type oméga-3. La présence de quantités relativement importantes d'acides gras oméga-3 est particulièrement intéressante dans ce contexte, compte tenu du rôle possible de ces nutriments, seuls (Hibbeln et al., 1998) ou en association avec d'autres nutriments (Gesch et al., 2002), dans la prévention et le traitement des comportements violents et des états dépressifs.

Tableau 2. Valeur nutritionnelle du Vitapoche ${ }^{\circledR}$

\begin{tabular}{|c|c|c|c|}
\hline & $\begin{array}{l}\text { Valeur nutritionnelle } \\
\text { pour un sachet de } 70 \mathrm{~g}\end{array}$ & & $\begin{array}{l}\text { Valeur nutritionnelle } \\
\text { pour un sachet de } 70 \mathrm{~g}\end{array}$ \\
\hline Energie totale & $377 \mathrm{kcal}$ & Potassium & $607 \mathrm{mg}$ \\
\hline Glucides $(33,3 \mathrm{~g} / 70 \mathrm{~g})$ & $133 \mathrm{kcal}$ soit 35,4\% de l'énergie totale & Magnésium & $65 \mathrm{mg}$ \\
\hline Lipides $(23,3 \mathrm{~g} / 70 \mathrm{~g})$ & 210 kcal soit $55,6 \%$ de l'énergie totale & $\operatorname{Zinc} *$ & $5 \mathrm{mg}$ \\
\hline Protides $(8,5 \mathrm{~g} / 70 \mathrm{~g})$ & 34 kcal soit 9,0\% de l'énergie totale & Vitamine B1 * & $14 \mathrm{mg}$ \\
\hline Acides gras saturés & $10,2 \mathrm{~g}$ & Niacine (vit PP)* & $18 \mathrm{mg}$ \\
\hline Acides gras monoinsaturés & $8,5 \mathrm{~g}$ & Acide folique (vit B9)* & $200 \mu g$ \\
\hline Acides gras polyinsaturés & $4,5 \mathrm{~g}$ & Vitamine $\mathrm{B} 12 *$ & $10 \mu g$ \\
\hline dont $C 18: 2 n-6$ & $3,2 \mathrm{~g}$ & Vitamine C* & $80 \mathrm{mg}$ \\
\hline$C 18: 3 n-3$ & $1,3 \mathrm{~g}$ & Vitamine D* & $5 \mu g$ \\
\hline Rapport n-6/n-3 & 2,4 & Vitamine $\mathrm{E} *$ & $5 \mathrm{mg}$ \\
\hline Calcium * & $800 \mathrm{mg}$ & & \\
\hline
\end{tabular}

* nutriment ayant fait l'objet d'un enrichissement.

Caractéristiques : Le Vitapoche ${ }^{\circledR}$ est une pâte fondante au cacao prête à consommer, emballée en sachets individuels de $70 \mathrm{~g}$.

Composition : Matière grasse végétale, poudre de lait écrémé, sucre, lactosérum, cacao, malto-dextrines, vitamines $\mathrm{C}, \mathrm{E}, \mathrm{D}$, thiamine, B12, niacine, acide folique, calcium, zinc, lécithine. Ne contient pas de substance d'origine animale, sauf ingrédients laitiers. 


\section{Le Vitapoche ${ }^{\circledR}$ : un aliment enrichi adapté à une utilisation dans la rue}

Le Vitapoche ${ }^{\circledR}$ est un aliment riche en énergie $(500 \mathrm{kcal} / 100 \mathrm{~g})$. Ceci représente un atout majeur pour une utilisation dans la rue. Tout d'abord, la forte densité énergétique permet d'avoir un sachet de petite taille, facile à glisser dans la poche. Ensuite, du fait de sa richesse en lipides, la pâte est fortement hydrophobe, ce qui lui confere une très bonne stabilité microbiologique (on n'observe aucun développement bactérien dans un sachet ouvert, même s'il a été contaminé volontairement et laissé ouvert à température ambiante pendant plusieurs jours) (Briend, 2001a). Enfin, la viscosité intermédiaire de la pâte facilite son ingestion par des personnes ayant une dentition incomplète, ce qui est fréquemment le cas des personnes sans-abri. Compte tenu du faible risque de surpoids (Darmon et al., 2001b; Darmon et al., 2002) et des faibles apports en lipides observés dans cette population (32\% et $22 \%$ de l'apport énergétique hors alcool respectivement dans l'étude INSERM (Darmon et al., 2001b) et dans celle du SAMU social (Malmauret et al., 2002)) par rapport à la population générale (de 39 à $40 \%$ selon les études (groupe de travail réuni par le Haut Comité de la Santé publique, 2000)), le fort pourcentage en lipides de la pâte $(55 \%)$ a été considéré comme acceptable par le Comité d'experts en nutrition humaine de l'Agence française de sécurité sanitaire des aliments (AFSSA), qui a délivré un avis favorable (publié le 31 janvier 2002) à son utilisation dans le cadre d'une étude d'acceptabilité, décrite ci-après.

\section{Le Vitapoche ${ }^{\circledR}$ : un aliment enrichi bien accepté par les personnes sans-abri}

L'acceptabilité du Vitapoche ${ }^{\circledR}$ a été testée auprès de la population cible dans le cadre d'une étude-action du PNNS coordonnée par la Direction générale de la santé (Darmon et al., 2003). L'étude a été réalisée en deux phases et a eu lieu dans huit structures partenaires. Une première phase s'est déroulée en hiver: 16000 sachets d'un aliment prototype ont été distribués et 253 personnes sans-abri ont été invitées à donner leur avis sur cet aliment. Les résultats de ces premières enquêtes ont permis d'améliorer la formulation et la présentation de l'aliment, et d'adapter la quantité de pâte par sachet de façon à ce qu'une consommation journalière d'un sachet par jour soit une hypothèse réaliste. Le Vitapoche ${ }^{\circledR}$ est actuellement disponible en sachets de $70 \mathrm{~g}$, car $27 \%$ des personnes interrogées avaient trouvé la quantité de $50 \mathrm{~g}$ insuffisante, contre seulement $7 \%$ non satisfaits des sachets de $70 \mathrm{~g}$. C'est aussi à l'issue de cette première phase que nous avons donné son nom définitif au Vitapoche ${ }^{\circledR}$, afin de tenir compte des remarques des personnes enquêtées à ce sujet. Quinze mille sachets d'une nouvelle production ont été distribués au cours de la seconde phase, en été, et 130 enquêtes réalisées. Les principaux résultats sont les suivants: quatre personnes sur cinq ont consommé du 
Vitapoche ${ }^{\circledR}$ le jour où elles en ont reçu; parmi celles qui en ont reçu plusieurs jours de suite, quatre sur cinq en ont effectivement consommé tous les jours; deux personnes sur trois ont déclaré qu'elles consommeraient régulièrement $\mathrm{du}$ Vitapoche ${ }^{\circledR}$ si elles pouvaient se le procurer facilement. Ces résultats suggèrent une bonne acceptabilité du Vitapoche ${ }^{\circledR}$ par la population cible, puisque, à l'exception de certains aliments tels que le pain, le lait, le beurre ou la confiture consommés au petit déjeuner, rares sont les aliments consommés de façon quotidienne par un pourcentage important de personnes, qu'ils soient transformés industriellement ou non. Les résultats de la seconde phase de l'étude d'acceptabilité ont permis d'améliorer le Vitapoche ${ }^{\circledR}$, notamment sur le plan de sa présentation, très proche dans sa version définitive de celle des autres aliments à base de chocolat actuellement disponibles dans le commerce. Ceci devrait permettre d'augmenter encore l'acceptabilité du produit. À l'issue de l'étude d'acceptabilité, la DGCCRF a délivré (le 12 août 2003) une autorisation de commercialisation du Vitapoche ${ }^{\circledR}$ dans le cadre du réseau associatif du secteur alimentaire et des centres de soin d'urgence.

\section{Le Vitapoche ${ }^{\circledR}$ : un double intérêt économique, pour le donateur et pour le bénéficiaire}

Dans l'enquête de l'INSERM, seulement $50 \%$ des sujets avaient dépensé de l'argent pour leur alimentation (aliments, y compris eau minérale, lait ou jus de fruits) au cours des dernières $48 \mathrm{~h}$. Chez ceux qui avaient dépensé de l'argent, les dépenses alimentaires s'élevaient en moyenne à $2,20 \quad € / j$ et diminuaient régulièrement au cours du mois (Darmon et al., 2000). Ces achats ne représentaient que $15 \%$ en moyenne des apports énergétiques hors alcool (Darmon et al., 2001b). Nous avons complété ces données d'achats avec des données similaires recueillies dans l'enquête d'acceptabilité du Vitapoche ${ }^{\circledR}$, afin de pouvoir établir une liste réaliste des aliments achetés dans la rue par les personnes sans-abri, et du prix payé en moyenne par ces personnes pour ces aliments. En faisant appel à la programmation linéaire, selon une méthodologie décrite précédemment (Briend, 2001b; Briend et al., 2003 ; Darmon et Briend, 2001a), nous avons ensuite calculé le prix théorique nécessaire pour obtenir une ration de $2400 \mathrm{kcal}$ respectant l'ensemble des ANC pour un homme adulte (i.e., équilibre convenable entre protéines, lipides et glucides, et apports adéquats en fibres, vitamines et minéraux) (Martin, 2001). Un apport énergétique journalier égal à $2400 \mathrm{kcal}$ a été choisi pour cette modélisation, car ceci correspond à la moyenne des apports énergétiques hors alcool pour les hommes dans la population générale (Volatier, 2000). Ceci correspond aussi au niveau qui serait atteint si on ajoutait un sachet de Vitapoche ${ }^{\circledR}$ à l'apport énergétique observé en moyenne dans l'enquête INSERM chez des hommes sans-abri hébergés en centre d'urgence. 
Figure 1. Impact du type d'aide alimentaire sur le coût minimal nécessaire pour respecter l'ensemble des ANC pour un homme adulte (Martin, 2001)

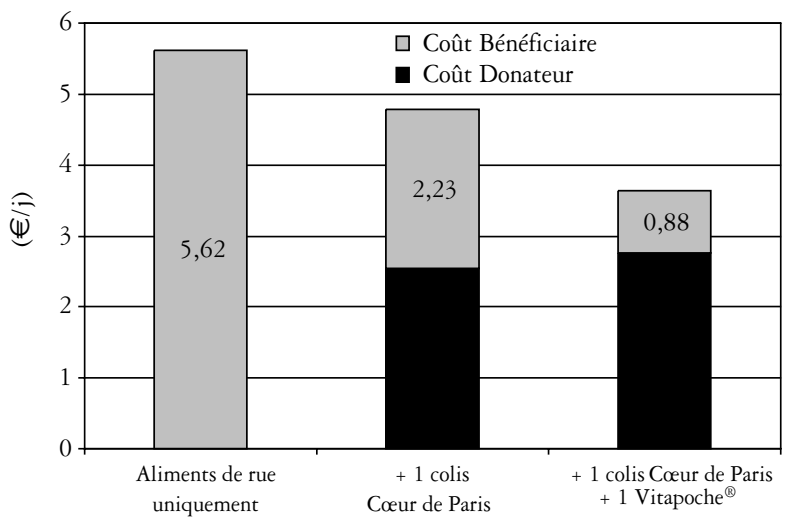

La figure 1 montre que le prix minimum théorique pour respecter l'ensemble des ANC varie selon la source d'approvisionnement :

- Quand uniquement des aliments achetés et consommés dans la rue sont disponibles, un strict minimum de $5,62 € / j$ est nécessaire pour respecter les ANC. Cette somme est nettement supérieure à la quantité d'argent dont dispose une personne sans-abri pour se nourrir. Elle est aussi bien supérieure aux 3,31 €/j calculés précédemment pour le respect des ANC dans le cas d'un accès normal à tous les aliments, avec la possibilité de les stocker et de les cuisiner (Darmon et al., 2006). Ceci démontre, si cela était nécessaire, que la réalisation de l'équilibre alimentaire dans la rue pose non seulement des problèmes pratiques évidents, mais représente aussi un véritable luxe sur le plan financier.

- L'accès à une aide alimentaire classique permet de diminuer ce coût journalier minimal. Les colis-repas du "Cour de Paris », le service d'aide alimentaire de la mairie de Paris, ont été considérés dans l'exemple présenté figure 1. Ces colis apportent $1200 \mathrm{kcal}$ en moyenne (Gendron et al., 2003). La figure montre qu'une personne recevant un tel colis devrait le compléter avec $2,23 € / j$ d'achat d'aliments « de rue » pour respecter l'ensemble des ANC.

- La situation la plus favorable est celle où le bénéficiaire reçoit ce même colisrepas complété d'un sachet de Vitapoche ${ }^{\circledR}$. Dans ce cas, l'aide alimentaire atteint une qualité nutritionnelle de très haut niveau, qui ne pourrait pas être obtenue en absence d'aliment enrichi. Il suffit de compléter ce don avec 0,88 € d'aliments achetés dans la rue pour pouvoir atteindre l'ensemble des ANC.

Ces chiffres sont théoriques et doivent être interprétés et utilisés avec précaution. L'analyse montre bien, cependant, le double intérêt économique du Vitapoche ${ }^{\circledR}$, non seulement pour le donateur (ajouter un sachet de Vitapoche ${ }^{\circledR}$ au colis ne lui coûte que $0,21 €)$, mais aussi pour le bénéficiaire, qui devra dépenser deux à trois fois moins pour compléter le colis reçu. On peut également démontrer l'intérêt 
économique et nutritionnel du Vitapoche ${ }^{\circledR}$ à d'autres niveaux d'apports énergétiques totaux (notamment $2700 \mathrm{kcal}$, correspondant aux ANC pour un homme adulte), et à d'autres niveaux de contribution de l'aide alimentaire à ces apports (résultats non montrés). De plus, nous avons choisi dans notre exemple une ration donnée de très bonne qualité nutritionnelle (Gendron et al., 2003). Si nous étions partis d'une ration moins bien équilibrée, l'intérêt nutritionnel et économique du Vitapoche ${ }^{\circledR}$ aurait été encore plus évident.

\section{Conclusion}

Le statut nutritionnel des personnes sans-abri pourrait être amélioré par une consommation régulière de Vitapoche ${ }^{\circledR}$, puisque celui-ci est spécifiquement enrichi en nutriments risquant d'être déficitaires dans leur ration. La première production industrielle de Vitapoche ${ }^{\circledR}$ a eu lieu en janvier 2004. La fourniture gratuite de Vitapoche ${ }^{\circledR}$ aux associations désireuses de le distribuer est une des propositions du PNNS pour les années 2006-2008. Ceci pourrait s'effectuer via les DDASS, par commande groupée départementale financée par le fonds national de promotion de la nutrition. Certaines associations ont cependant exprimé des réticences quant à l'utilisation de cet aliment enrichi, celui-ci étant perçu comme un substitut de repas pouvant dispenser du lien social inhérent au repas partagé (Serafini, 2003). Il apparaît donc important de souligner que le Vitapoche ${ }^{\circledR}$ n'est pas un substitut de repas. Chaque sachet apporte $377 \mathrm{kcal}$, ce qui correspond en moyenne à la quantité d'énergie contenue dans une barre chocolatée, mais pas à celle d'un vrai repas. Il est donc destiné, non pas à remplacer l'aide alimentaire déjà existante, mais à en optimiser la qualité nutritionnelle. Conçu dans une optique de prévention nutritionnelle, le Vitapoche ${ }^{\circledR}$ doit être intégré à une démarche globale d'accompagnement des personnes, pour une meilleure prise en charge de leur santé. Son acceptabilité est meilleure s'il est proposé dans le cadre d'un dialogue et accompagné d'informations, sur l'équilibre et l'hygiène alimentaires notamment. C'est une des raisons pour lesquelles le Vitapoche ${ }^{\circledR}$ n'est pas disponible dans le commerce comme les autres aliments.

Le Vitapoche ${ }^{\circledR}$ devrait se substituer, sans les supprimer totalement, à des aliments du type barres chocolatées, chips et gâteaux secs, souvent consommés par les personnes sans-abri, car ils sont bons, pratiques et économiques. Tout en restant pratique et agréable à consommer, le Vitapoche ${ }^{\circledR}$ a une bien meilleure qualité nutritionnelle que ces aliments et représente une option économique pour le donateur. L'introduction de Vitapoche ${ }^{\circledR}$ dans les programmes d'aide alimentaire destinés aux personnes en situation de grande exclusion apparaît, dans le contexte actuel, comme le moyen le plus efficace pour améliorer leur statut vitaminique et minéral. Ceci pourrait contribuer à prévenir des pathologies nutritionnelles spécifiques, telles que le scorbut, la pellagre ou l'encéphalopathie de Wernicke, et limiter le recours à des suppléments nutritionnels inadaptés. 


\section{Bibliographie}

Babidge N.C., Buhrich N. and Butler T. (2001). Mortality among homeless people with schizophrenia in Sydney, Australia : A 10-year follow-up, Acta Psychiatrica Scandinavia, 103, pp. 105-10.

Barrow S.M., Herman D.B., Córdova P. and Struening E.L. (1999). Mortality among homeless shelter residents in New York City, American Journal of Public Health, 89, pp. 529-34.

Briend A. (2001a). Highly nutrient-dense spreads: A new approach to delivering multiple micronutrients to high-risk groups, British Journal of Nutrition, 85, Suppl 2, S175-S179.

Briend A., Darmon N., Ferguson E. and Erhardt J.G. (2003). Linear programming: A mathematical tool for analyzing and optimizing children's diets during the complementary feeding period, Journal of Pediatry Gastroenterology and Nutrition, 36, pp. 12-22.

Briend A., Ferguson E. and Darmon N. (2001b). Local food price analysis by linear programming: A new approach to assess the economic value of fortified food supplements, Food Nutrition Bulletin, 22, pp. 184-9.

Brousse C., La Rochère B. de et Massé E. (2002). Hébergement et distribution de repas chauds. Le cas des sans-domicile, INSEE première, 823, pp. 1-4.

Cadau M., Convers J.-P., Bonneau C., Mathias C. et Chamson A. (2001). Statut vitaminique de sujets en état de précarité, 5 ième Symposium sur les Vitamines et les Biofacteurs, 8-10 février.

Cook C.C., Hallwood P.M. and Thomson A.D. (1998). B Vitamin deficiency and neuropsychiatric syndromes in alcohol misuse, Alcohol Alcohol, 33, pp. 317-36.

Cross C.E., Halliwell B. (1993). Nutrition and human disease: How much extra vitamin C might smokers need?, The Lancet, 341, p. 1091.

Dalton K., Dalton M.J. (1987). Characteristics of pyridoxine overdose neuropathy syndrome, Acta Neurologica Scandinavia, 76, pp. 8-11.

Darmon N., Briend A. (2001a). Consommations alimentaires, ANC et modélisation: utilisation de la programmation linéaire, in: Apports nutritionnels conseillés pour la population française, Martin A. (éd.), Paris, Lavoisier, pp. 452-7.

Darmon N., Coupel J., Deheeger M. and Briend A. (2001b). Dietary inadequacies observed in homeless men visiting an emergency night shelter in Paris, Public Health Nutrition, 4, pp. 155-61.

Darmon N., Coupel J., Deheeger M. et Briend A. (2000). L'alimentation des SDF : des risques possibles pour la santé ? Alimentation et Précarité, 9, pp. 7-9. 
Darmon N., Ferguson E. and Briend A. (2006). Impact of a cost constraint on nutritionally adequate food choices for French women: An analysis by linear programming, Journal of Nutrition Education and Behavior, 38, pp. 82-6.

Darmon N., Lécossais C. et Briend A. (2003). Comment améliorer l'aide alimentaire destinée aux personnes sans-abri ? Acceptabilité d'un aliment de rue enrichi, Médecine et Nutrition, 39, pp. 15-22.

Darmon P., Samouda H., Guillaume V., Dadoun P., Raoult D. et Brouqui P. (2002). Evaluation anthropométrique de l'état nutritionnel d'une population de sujets sans domicile fixe à Marseille, Nutrition Clinique et Métabolique, 16 (Suppl. 1), 39 S.

Darnton-Hill I., Truswell A.S. (1990). Thiamin status of a sample of homeless clinic attenders in Sydney, Medical Journal Australia, 152, pp. 5-9.

Derrickson J.P., Widodo M.M. and Jarosz L.A. (1994). Providers of food to homeless and hungry people need more dairy, fruit, vegetable, and lean-meat items, Journal of The American Dietetic Association, 94, pp. 445-6.

Diop E.H., Dossou N.I., Ndour M.M., Briend A. and Wade S. (2003). Comparison of the efficacy of a solid ready-to-use food and a liquid, milk-based diet for the rehabilitation of severely malnourished children : A randomized trial, American Journal of Clinical Nutrition, 78, pp. 302-7.

Fain O., Thomas M. (1997). Le scorbut actuellement, Cabiers de Nutrition et de Diététique, 32, pp. 300-5.

Gelberg L., Stein J.A. and Neumann C.G. (1995). Determinants of undernutrition among homeless adults, Public Health Reports, 110, pp. 448-54.

Gendron S., Pérochon P. et Darmon N. (2003). Qualité nutritionnelle de colis alimentaires distribués à des personnes sans-abri à Paris, Information diététique, 2 , pp. 9-12.

Gesch C.B., Hammond S.M., Hampson S.E., Eves A. and Crowder M.J. (2002). Influence of supplementary vitamins, minerals and essential fatty acids on the antisocial behaviour of young adult prisoners. Randomised, placebo-controlled trial, British Journal of Psychiatry, 181, pp. 22-8.

Groupe de travail réuni par le Haut Comité de la Santé publique (2000). Consommation alimentaire et état nutritionnel de la population vivant en France, in: Pour une politique nutritionnelle de santé publique en France. Enjeux et propositions, Collection Avis et Rapports, Rennes, pp. 189-95.

Hibbeln J.R., Umhau J.C., Linnoila M., George D.T., Ragan P.W., Shoaf S.E., Vaughan M.R., Rawlings R. and Salem N. Jr. (1998). A replication study of violent and nonviolent subjects : Cerebrospinal fluid metabolites of serotonin and dopamine are predicted by plasma essential fatty acids, Biology and Psychiatry, 44, pp. 243-9. 
Hwang S.W. (2001). Homelessness and health, Canadian Medical Association Journal, 164, pp. 229-33.

Hwang S.W. (2000). Mortality among men using homeless shelters in Toronto (Ontario), Journal of the American Medical Association, 283, pp. 2152-7.

Hwang S.W., Lebow J.M., Bierer M.F., OConnell J.J., Orav E.J. and Brennan T.A. (1998). Risk factors for death in homeless adults in Boston, Archives of Internal Medicine, 158, pp. 1454-60.

Jacobs-Starkey L. (1994). An evaluation of emergency food bags, Journal of the Canadian Dietetic Association, 55, pp. 175-8.

Kanis J., Johnell O., Gullberg B., Allander E., Elffors L., Ranstam J., Dequeker J., Dilsen G., Gennari C., Vaz A.L., Lyritis G., Mazzuoli G., Miravet L., Passeri M., Perez C.R., Rapado A. and Ribot C. (1999). Risk factors for hip fracture in men from southern Europe: the MEDOS (Mediterranean Osteoporosis) Study, Osteoporosis International, 9, pp. 45-54.

Kertesz S.G. (2001). Pellagra in two homeless men, Mayo Clinic Proceedings, 76, p. 318.

Langnase K., Muller M.J. (2001). Nutrition and health in an adult urban homeless population in Germany, Public Health Nutrition, 4, pp. 805-11.

Laven G.T., Brown K.C. (1985). Nutritional status of men attending a soup kitchen: A pilot study, American Journal of Public Health, 75, pp. 875-8.

Lecomte T., Mizrahi A. (1997). Recours aux soins et morbidité des personnes sans domicile permanent en région parisienne, Bulletin de l'Académie nationale de Médecine, 181, pp. 1715-27.

Lecomte E., Herbeth B., Pirollet P., Chancerelle Y., Arnaud J., Musse N., Paille F., Siest G. and Artur Y. (1994). Effect of alcohol consumption on blood antioxidant nutrients and oxidative stress indicators, American Journal of Clinical Nutrition, 60, pp. 255-61.

Luder E., Boey E., Buchalter B. and Martinez-Weber C. (1989). Assessment of the nutritional status of urban homeless adults, Public Health Reports, 104, pp. 451-7.

Luder E., Ceysens-Okada E., Koren-Roth A. and Martinez-Weber C. (1990). Health and nutrition survey in a group of urban homeless adults, Journal of the American Dietetic Association, 90, pp. 1387-92.

Malmauret L., Leblanc J., Cuvelier I. and Verger P. (2002). Dietary intakes and vitamin status of a sample of homeless people in Paris, European Journal of Clinical Nutrition, 56, pp. 313-20.

Marpsat M. (1999). Les sans-domicile à Paris et aux États-Unis, in : Données sociales : la société française, Paris, INSEE, pp. 480-9. 
Martin A. (2001). Apports nutritionnels conseillés pour la population française, Paris, Lavoisier.

Ministère de la Santé (2001). Programme national Nutrition Santé, http:// www.sante.gouv.fr/htm/pointsur/nutrition/index.htm

Robertson M.J., Cousineau M.R. (1986). Health status and access to health services among the urban homeless, American Journal of Public Health, 76, pp. 561-3.

Roy E., Boivin J.F., Haley N. and Lemire N. (1998). Mortality among street youth, The Lancet, 352, p. 32.

Rushton C., Wheeler E. (1993). The dietary intake of homeless males sleeping rough in Central London, Journal of Human Nutrition and Dietetics, 6, pp. 443-56.

Salit S.A., Kuhn E.M., Hartz A.J., Vu J.M. and Mosso A.L. (1998). Hospitalization costs associated with homelessness in New York City, New England Journal of Medicine, 338, pp. 1734-40.

Serafini T. (2003). Le repas, un moyen de créer un lien, Libération, 16 décembre.

Shanks N.J. (1988). Medical morbidity of the homeless, Journal of Epidemiology Community and Health, 42, pp. 183-6.

Shaw M., Dorling D. (1998). Mortality among street youth in the UK, The Lancet, 352, p. 743 .

Silliman K., Yamanoha M.M. and Morrissey A.E. (1998). Evidence of nutritional risk in a population of homeless adults in rural northern California, Journal of the American Dietetic Association, 98, pp. 908-10.

Takano T., Nakamura K., Takeuchi S. and Watanabe M. (1999). Disease patterns of the homeless in Tokyo, Journal of Urban Health, 76, pp. 73-84.

Terrolle D. (2002). La mort des SDF à Paris : un révélateur social implacable, Etudes sur la mort, 122, p. 68.

Volatier J.-L. (coord.) (2000). Enquête INCA (individuelle et nationale sur les consommations alimentaires), publiée à l'initiative de l'AFSSA, de la DGAL et du CREDOC, AFSSA (éd.), Paris, Lavoisier.

Vredevoe D.L., Brecht M.L., Shuler P. and Woo M. (1992). Risk factors for disease in a homeless population, Public Health Nursery, 9, pp. 263-9.

Wolgemuth J.C., Myers-Williams C., Johnson P. and Henseler C. (1992). Wasting malnutrition and inadequate nutrient intakes identified in a multiethnic homeless population, Journal of The American Dietetic Association, 92, pp. 834-9. 